Apidologie, 1972, 3 (2), 167-186.

\title{
ÜBER DIE STELLUNG VON ASPIDOSMIA BRAUNS 1926 NEBST ALLGEMEINEN ERÖRTERUNGEN DER PHYLOGENETISCHEN SYSTEMATIK DER MEGACHILIDE (INSECTA, HYMENOPTERA, APOIDEA).
}

Sur la position d'Aspidosmia Brauns 1926 et discussion générale de la systématique phylogénique des Megachilidae (Insecta, Hymenoptera, Apoidea).

Dieter Stefan PETERS

Forschungsinstitut und Naturmuseum

Senckenberg, Frankfurt am Main

\section{SUMMARY}

on the position of Aspidosmia Brauns 1926 With remarks ON PHYLOGENETIC SYSTEMATICS OF THE MEgACHILIDAE

(INSECTA, HYMENOPTERA, APOIDEA)

1. For the first time there is given a more detailed description of Aspidosmia. Only two species from South-Africa are known to belong to this genus : Aspidosmia arnoldi Brauns 1926 and $A$. volkmanni (FrIEse 1909). Osmia grobbelaari Brauns 1926 is regarded as the male of $A$. volkmanni. The original descriptions of $A$. arnoldi and $A$. volkmanni are completed and corrected. Aspidosmia seems to belong rather to the Anthidiinae than to Osmia. It can be regarded as the most primitive group (scopa on the sternits and on the hind legs ?) among the Anthidiinae.

2. For the Megachilidae there is offered a scheme of synapomorphies (see dendrogram). Fideliidae proved to be most likely the sister-group of the Megachilidae. Based on available data there is sketched a character-complex for the presumable ancestral species of the Megachilidae. 


\section{ZUSAMMENFASSUNG}

1. Aspidosmia wird erstmals genauer charakterisiert. Bisher sind zwei Arten dieser Gattung bekannt : Aspidosmia arnoldi BraUns 1926 und $A$. volkmanni (FrIEse 1909). Beide leben in Südafrika. Osmia grobbelaari BraUNS 1926 wird als Synonym von $A$. volkmanni betrachtet. Die Beschreibungen der beiden Aspidosmia-Arten werden ergänzt und berichtigt. Aspidosmia ist am ehesten zu den Anthidiinae zu stellen, wo sie als besonders ursprüngliche Gruppe (Scopa auch an den Hinterschienen?) allen anderen Anthidiinae gegenübersteht.

2. Für die Megachilidae wird ein Synapomorphieschema entworfen (s. Stammbaum). Als Schwestergruppe der Megachilidae sind die Fideliidae anzusehen. Aufgrund der Befunde wird ein kurzes Merkmalsschema für die Stammart der Megachilidae zusammengestellt.

\section{A. - EINLEITUNG}

Brauns wollte Aspidosmia als Untergattung von Osmia verstanden wissen. Allerdings führte er den Namen nur in der Überschrift zu der Beschreibung seiner Osmia arnoldi und in der Unterschrift zu den dazugehörigen Abbildungen an. Eine Kennzeichnung im strengen Sinne und eine Abgrenzung gegen andere Untergattungen von Osmia fehlt. Die Aufstellung der neuen Untergattung wurde also auf der Beschreibung von Osmia arnoldi begründet, letztere ist somit als Typus-Art von Aspidosmia anzusehen.

Inzwischen wurde eine weitere Art gefunden, die fraglos ebenfalls zu Aspidosmia gezählt werden muß, so daß sich nunmehr die Gruppe genauer kennzeichnen läßt. Die Untersuchung dieser äußerst merkwürdigen Bienen zeitigte einige Ergebnisse, die die Stellung von Aspidosmia problematisch machen und außerdem für die phylogenetische Betrachtung der gesamten Megachiliden bedeutsam erscheinen.

\section{B. - DIE ARTEN UND DIE KENNZEICHEN VON ASPIDOSMIA}

Aspidosmia arnoldi

1926 Osmia (subgen. Aspidosmia) arnoldi oै $\uparrow-$ H. Brauns, Zool. Jahrbuch, Syst. 52 : 208-212, Fig. 5 a-c.

Die umfangreiche Beschreibung läßt darauf schließen, daß Brauns von dem bizarren Aussehen dieser Biene recht beeindruckt war. Vor allem der Kopf des Weibchens mit seiner Clypeusplatte, den weitausladenden Mandibeln und den langen Zapfen der Postgenae wirkt wahrhaftig monströs (Abb. 1). Dadurch gefesselt, hat Brauns wohl einige andere bemerkenswerte Eigenheiten übersehen. Die Beschreibung mag deshalb hier ergänzt werden.

₹ : Maxillarpalpen fünfgliedrig. Die Länge der Glieder in der Reihenfolge 3, 4, 2, 1, 5 abnehmend. Der Kaurand der Mandibeln ist nur von außen betrachtet dreizähnig; auf der Innenfläche, etwa hinter dem obersten Zahn, und von diesem teilweise verdeckt, befindet sich noch ein vierter. Die Basis des Labrum ist von dessen Rest durch eine kräftige, mitten unterbrochene Querleiste getrennt. Strigilis der Protibia in eine lange dornförmige, am Rand gezähn- 
elte Spitze ausgezogen. Hinterhüften ohne ventrale Längsleiste. Hinterschienen außen gleichmäßig mit langen, ungefiederten Haaren bedeckt, so daß der Eindruck einer Scopa entsteht. Die Ansatzstellen der einzelnen Haare hier knötchenförmig erhaben, wodurch die Oberfläche der Schiene rauh und holperig ist. Krallen der Klauenglieder gespalten. Flügelbehaarung wenig differenziert, lediglich randwärts kürzer und dichter werdend. Jugallapen des Hinterflügels annähernd halb so lang wie Anallapen. Flügelmal an seinem Hinterrand (in der 1. Submarginalzelle $=1$. Cubitalzelle) kürzer als seine größte Breite. Tergite 3-5 in ihrem letzten Viertel eingedrückt, der glatte, punktlose Rand dagegen wieder etwas aufwärts gebogen erscheinend. Eine ähnliche Bildung bei Tergit 2 angedeutet. Tergit 1 mit einer langen Naht entlang des seitlichen Knicks. Im Gegensatz zur Angabe bei BraUns sind auch die Tergite zwar nur mäßig dicht, aber nicht “ fein und kurz », sondern ziemlich lang und eher grob behaart. Das ganze Tier macht einen struppigen, “ unordentlichen » Eindruck.

* : Gesicht viel breiter als lang. Labrumbasis mit mitten nnterbrochenem Querwulst. Clypeusrand überragt nicht die Labrumbasis. Maxillarpalpen viergliedrig (nur bei einem Exemplar nachgeprüft). Kaurand der Mandibeln Abb. 3. Clypeus an der Basis aufgebläht, seine distale Hälfte mit einem breiten gelben Querband, das wohl meistens von der dichten Behaarung verdeckt ist. Fühlerglieder 3-5 ventral mit einem schwach glänzenden Längswulst. Die Fühler reichen, entgegen der wohl irrtümlichen Angabe bei Brauns, nicht bis zum Vorderrand, sondern fast bis zum Hinterrand des Thorax. Flügel deutlich schwächer behaart als beim Weibchen. Tergitbildung ähnlich wie beim Weibchen. Tergit 7 breit, mit geradem Endrand, der in der Mitte anscheinend schwächer sklerotisiert ist. Dieses Tergit mit langen, schwarzen, borstenförmigen, glänzenden Haaren, die zur Mitte gebogen sind und sich hier teilweise überkreuzen. Sternit 7 und 8 sowie Genitale wie auf Abb. 4-6. Sternit 8 mit ziemlich langen Haaren am Rand. Diese Haare zeigen etwas über der Mitte eine hellere Zone, an der sie leicht abbrechen. Sternit 6 seicht V-förmig ausgeschnitten, in der Mitte zusätzlich tiefer eingekerbt. Bei dem präparierten Exemplar waren Sternit und Tergit 8 mit ihren seitlichen Fortsätzen fest miteinander verwachsen. Tergit 8 war nur noch ein stark reduziertes häutiges Gebilde.

Brauns zog diese absonderliche Biene aus einem Nest, das aus c zerkauter Pflanzenmasse » und « kleinen Steinchen » an Steine gemauert war. Bemerkenswert ist die Angabe : c Dasselbe Nest ergab 4, Jahre hintereinander einige Bienen, obgleich es isoliert und eingezwingert war. ”

Mir lagen 7 o und $2 \sigma^{\star}$ vor, alle von Willowmore, Capland. Da ich noch nicht sämtliche Syntypen gesehen habe, soll ein Lectotypus später festgelegt werden. Die Belegstücke entstammen dem Transvaal Museum, Pretoria, dem Albany Museum, Grahamstown, und dem National Museum of Southern Rhodesia, Bulawayo.

Aspidosmia volkmanni

1909 - Osmia volkmanni 우 - H. Friese, Die Bienen Afrikas, in : Jenaische Denkschriften $14: 320$.

1926 - Osmia grobbelaari o - A. Brauns, Zool. Jahrb. Syst. 52 : 217-218 (nov. syn.).

FrIESE vergleicht in seiner kurzen Beschreibung diese Biene mit O. fulviventris. Die Ähnlichkeit besteht jedoch nur oberflächlich.

Die Beschreibung sei hier ergänzt.

o : Labrumbasis mit einem Querwulst. Diese Basis wird vom Clypeus nicht überragt. Letzterer ist bauchig gebläht, sein Rand seicht konkav. Strigilis der Vordertibien wie bei arnoldi, Dorn eher noch etwas länger. Alle Klauen gespalten. Hinterschienen mit gelblicher Scopa-ähnlicher Behaarung. Oberfläche der Schiene wie bei arnoldi gekörnt. Der innere Sporn dieser Schienen lang und schwach nach außen gebogen, der äußere kürzer und in seinem letzten Viertel auffällig nach hinten gebogen. Flügelmal am Innenrand etwa so lang wie seine größte 

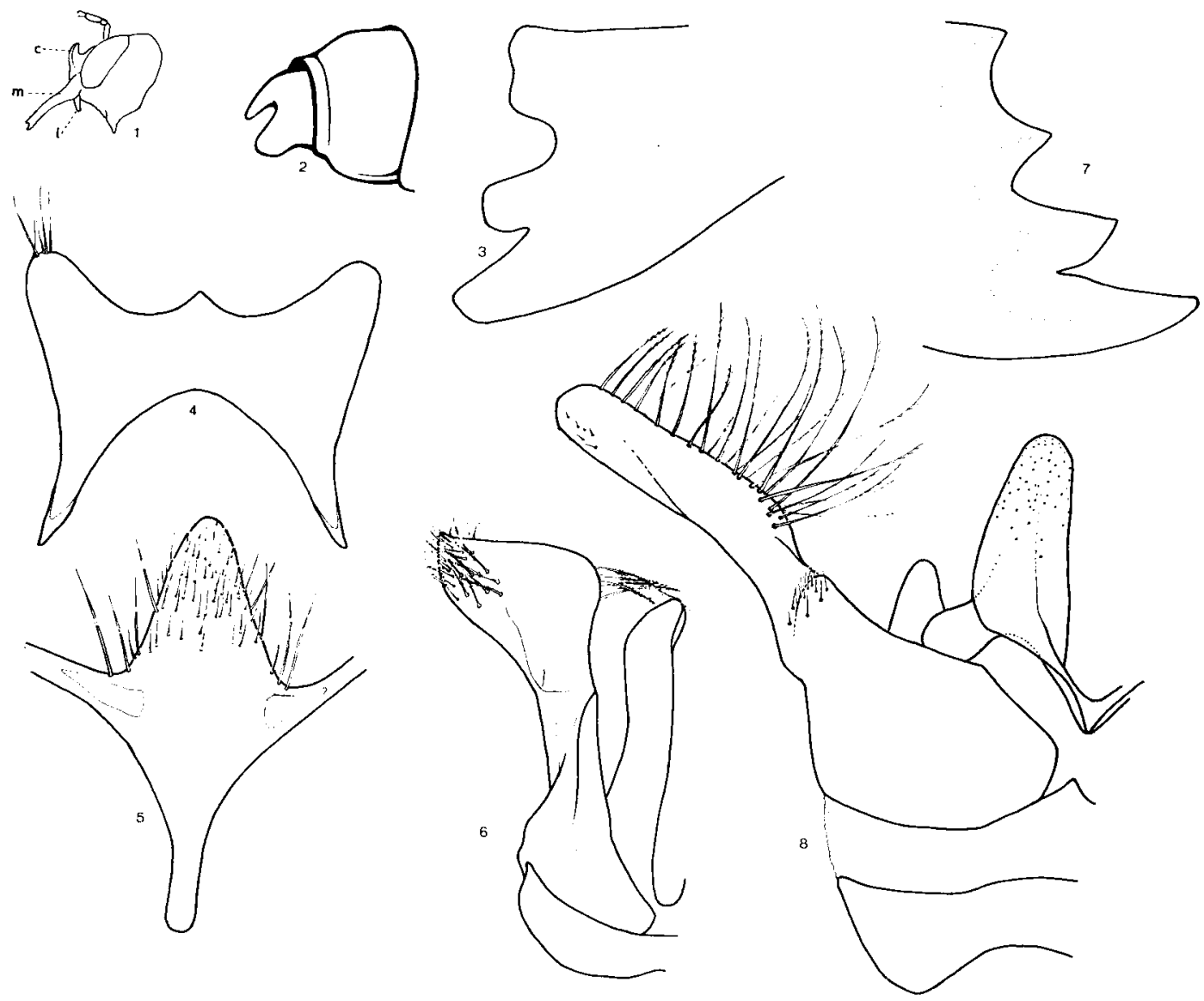

Aвв. 1-8. - 1. Aspidosmia arnoldi o, Kopf von links, c - Clypeus, 1 - Labrum, m - Mandibel, Fühler unvollständig, 2. Aspidosmia volkmanni d, Hinterleibsende, schräg von rechts hinten, das letzte Tergit etwas herausgezogen; in " Normalstellung 》 liegt seine abwärts gerichtete, mitten ausgeschnittene Fläche etwa in der Ebene des Hinterrandes des davorliegenden Tergits; Behaarung nicht gezeichnet. 3.-6. Aspidosmia arnoldi ơ. 3. Kaurand der linken Mandibel (leicht abgenutzt). 4. Siebentes Sternit. 5. Achtes Sternit 6. Genitale, linke Hälfte dorsal. 7.-8. Aspidosmia volkmanni đ. 7. Kaurand der rechten Mandibel!! 8. Genitale, linke Hälfte dorsal; in gequollenem Zustand gezeichnet, dadurch Parameren abgespreizt. - 1 . nach Brauns 1926, Fig. 5b;2.-8. Original. 3.-8. im gleichen Maßstab, 1. und 2. schwächer vergrößert.

Frg. 1-8. - 1. Aspidosmia arnoldi + , Tête vue de gauche. $c:$ clypeus. $l:$ labre. $m:$ mandibule, antenne incomplète.

2. Aspidosmia volkmanni đ, extrémité postérieure de l'abdomen, vue oblique de droite, en bas, le dernier tergite légèrement sorti; en « position normale » sa surface dirigée vers le bas et découpée au milieu se trouve à peu près dans le plan du bord postérieur du tergite précédent; pilosité non figurée.

3-6. Aspidosmia arnoldi ${ }^{\circ}$.

3 - Bord masticatoire de la mandibule gauche (légèrement usée).

4 - Septième sternite.

5 - Huitième sternite.

6 - Genitalia, moitié gauche, dorsale.

7-8. Aspidosmia volkmanni $\delta$.

7 - Bord masticatoire de la mandibule droite.
8 - Genitalia, moitié gauche, dorsale; dessinée à l'état turgescent, de telle sorte que les paramères sont saillantes.

1 : d'après Brauns 1926, Fig. $5 b$.

2-8 : figures originales,

3-8 : figures à la même échelle,

$1-2$ : grossissement plus faible. 
Breite. Mittelfeld des Propodeum groß und spiegelglatt; Propodeum seitlich davon ebenfalls glatt, aber mäßig dicht punktiert. Hinterleib relativ länger und schmaler als bei arnoldi, aber Tergite ebenfalls glatt und unregelmäßig ziemlich zerstreut punktiert (Zwischenräume punktgroß bis mehrfach größer). Tergite 3-5 am Ende deutlich eingedrückt, die glatten, bräunlichen Ränder ganz leicht aufgebogen erscheinend. Tergit 6 mitten schmal ausgeschnitten. Die gelbliche Behaarung der Tergite auf 3-5 mit längeren borstenartigen, schwarzen Haaren vermischt, Tergit 6 gleichmäßig kurz braungelb behaart.

\& : Erinnert sehr an arnoldi, und es erscheint merkwürdig, daß BrauNs dies nicht bemerkt hat. Gesicht etwas breiter als lang. Zu berichtigen ist die Angabe über die Mandibel, die nicht drei-, sondern vierzähnig ist (Abb. 7); zwischen den Zähnen 3 und 4 und undeutlicher zwischen 2 und 3 befindet sich ein eingedrückter schneidenartiger Rand. Der Clypeus zeigt eine gelbe Querbinde wie bei arnoldi. Seine stärkste Wölbung ist nicht an der Basis, sondern mehr in der Mitte. Die Fühler sind ohne besondere Kennzeichen und kürzer als bei jenem. Glied 3 ist gut doppelt so lang wie am Ende breit. Strigilis wie beim Weibchen, ebenso Sporen der Hinterschienen. Das ausgeschnittene Tergit 7 mit auffallend langen, borstenförmigen Haaren, die aber nicht so dick wie bei arnoldi sind. Die Abbildung der letzten Tergite bei Brauns ist nicht ganz treffend. Tergit 7 ist in Wirklichkeit stärker ausgebildet, seine distale Hälfte ist im Verhältnis zur Basis deutlich nach unten gebogen (Abb. 2). Der Gradulus von Tergit 6 biegt an den Seiten nach hinten um und bildet hier eine scharfkantige Leiste, die jedoch den Endrand nicht erreicht. Sternit 5 flach V-förmig ausgeschnitten, 6 ähnlich, aber flacher und in der Mitte in einen ventrad gerichteten Haken ausgezogen. Sternit 7 ähnlich wie bei arnoldi, aber noch kräftiger, die Seitenecken dornartig ausgezogen, das ganze Sternit mitten der Länge nach eingedrückt. Genitale wie Abb. 8. Der nicht abgebildete häutige Penis ist bemerkenswerterweise nur am Grund mit den Penisvalven verwachsen und in seinen Seitenwänden selbst leicht sklerotisiert. Die Valven haben deshalb viel mehr den Charakter von freistehenden « Sagitten » als dies gewöhnlich bei Megachiliden der Fall ist (vgl. SNodgrass 1941). Thorax und Hinterleib sonst sehr ähnlich wie beim Weibchen.

Die Artgleichheit von volkmanni und grobbelaari scheint mir außer Zweifel zu stehen. Beide ähneln den jeweils entsprechenden Geschlechtern von arnoldi. Die Übereinstimmung der Skulptur und Behaarung sowie der Hinterschienensporen bei volkmanni und grobbelaari machen die Entscheidung, beide für konspezifisch zu halten, nicht sehwer, zumal auch die Fundorte für die Zusammengehörigkeit sprechen. Friese scheint bereits zu demselben Schluß gekommen zu sein. In Berlin befindet sich nämlich ein Paar, dessen beide Geschlechter als Osmia volkmanni bezettelt sind. Das Männchen trägt sogar einen roten Typus-Zettel, der aber wohl nur irrtümlich dort angebracht ist und eigentlich dem Weibchen zukommt, das allein der Beschreibung zugrunde lag. Meines Wissens hat Friese eine Beschreibung des volkmanni- $\sigma^{*}$ nie veröffentlicht.

Belegstücke : 1 , S.W.-Afrika, Grootfontein 1903, Volkmann leg. (Holotypus); 1 o Klein-Nama, Ookiep, X. 1900 (Determination aber erst 1910 oder später), mit Typus-Schild (sicher Irrtum); beide Stücke im Zoologischen Museum, Berlin. 1 \& Ookiep, 9. 1890, R.M.L. leg. (Determination von Friese), South-African-Museum, Cape Town. 1 , Ookiep, Capland (Holotypus von O. grobbelaari, Typus No. 547), Transvaal-Museum, Pretoria.

Zusammenfassend können wir für Aspidosmia folgende besonders deutliche Merkmale feststellen :

a) Thorax annähernd kugelförmig, Metanotum liegt völlig in der abschüssigen Hinterwand. 
b) Parapsidalfurche des Mesonotum linienförmig.

c) Mittelfeld des. Propodeum glatt, nur ganz seitlich schwach gerunzelt. Auch an der Grenze zum Metanotum glatt und gerade. Propodeum seitlich des Mittelfeldes glatt, aber punktiert.

d) Flügelmal relativ kurz.

e) Basalnerv proximal gebogen (ähnlich, aber schwächer als bei Halictus), so daß er fast rechtwinklig auf den Cubitus (Medialnerv nach SchmieDEKNECHT) trifft. (Basalnerv und Nervulus interstitial, aber bei arnoldi क Nervulus ( postfurcal »).

f) Zwei Submarginalzellen. Die rücklaufenden Nerven münden in die zweite.

g) Jugallappen der Hinterflügel etwa von halber Länge des Anallappens.

h) Behaarung der Flügel wenig differenziert.

i) Strigilis der Vordertibien mit langer, gezähnelter Spitze.

j) Klauen gespalten.

k) Arolia vorhanden.

l) Hinterschienen der $\odot$ mit rauher, gekörnter Oberfläche und Scopa-ähnlicher Behaarung. (Bei dem volkmanni-Weibchen von Ookiep befinden sich in dieser Behaarung reichliche Pollenmassen, die den Eindruck machen, daß sie nicht nur zufällig oder beiläufig dorthin gelangt sind, zumal die Bauchscopa nicht sonderlich beladen ist (Abb. 9). Dieser

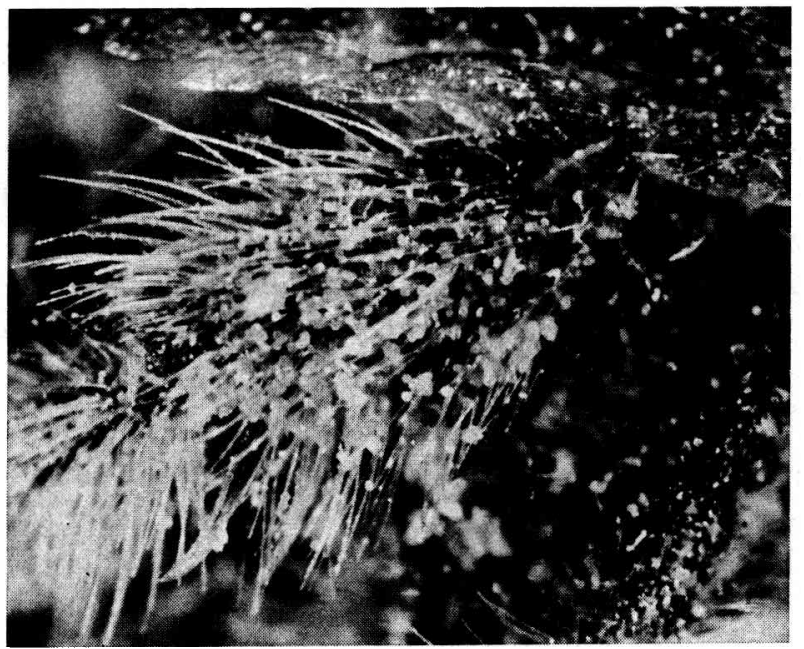

Aвв. 9. - Aspidosmia volkmanni, Scopa der rechten Hinterschiene mit Pollenladung. Zur Orientierung : Das Bein ist geknickt. Rechts unten ist der Trochanter, darüber der Femur zu sehen. Das Gelenk zwischen Schenkel und Schiene liegt etwa in der rechten oberen Bildecke und ist durch die Flügelmembran verdeckt. - Aufn. Senck. Museum (Haupt).

FIc. 9. - Aspidosmia volkmanni. Brosse du tibia postérieur droit avec charge de pollen. Pour information : la patte est pliée. En bas à droite se trouve le trochanter, au-dessus le fémur est visible. L'articulation entre la cuisse et le tibia se trouve à peu près dans le coin en haut et à droite de l'image et elle est recouverte par la membrane alaire. Photo. Senck. Museum (Haupt). 
Befund läßt den sehr starken Verdacht aufkommen, daß bei diesen Bienen Pollen auch an den Hinterbeinen eingetragen wird. Die vorliegenden Weibchen von arnoldi können leider zu diesem Punkt nichts beitragen, da sie von Brauns aus einem Nest gezogen wurden und deshalb keine Pollenladungen tragen. Ebenso scheint es sich bei dem Holotypus von volkmanni um ein ganz frisches Tier zu handeln, dessen Bauch und Beine pollenfrei sind.)

m) Hinterleibstergite zerstreut punktiert, mit glatten, eingedrückten, teilweise schwach aufgeworfenen Rändern.

n) Männchen mit 7 sichtbaren Tergiten.

o) Sternit 7 der Männchen gut entwickelt.

p) Männliches Genitale mit ventrad gebogenen Penisvalven.

q) Clypeus der Männchen mit einem gelben Querband, das aber nirgends den Clypeusrand erreicht.

r) Behaarung mäßig dicht, aber struppig, Haare hauptsächlich schmutzig gelbgrau und braunschwarz gefärbt, (bei den Männchen von arnoldi weißlich statt gelbgrau). Die schwarze Behaarung auf den letzten Tergiten teilweise borstenartig, bei den Männchen auf Tergit 7 stark entwickelt.

Vergleicht man die beiden Arten, so bemerkt man Unterschiede besonders am Kopf der Weibchen und an den Mandibeln, Fühlern und Genitalien der Männchen. Die männlichen Genitalien erscheinen auf den ersten Blick sogar außerordentlich verschieden zu sein. Bei arnoldi sind die Parameren kurz und gedrungen, ihr Ende ist schaufelartig erweitert. Bei volkmanni dagegen hat dieses Gebilde eine langgestreckte, zapfenartige Form. Bei näherer Betrachtung allerdings zeigen sich einige Ähnlichkeiten, die wahrscheinlich als Homologien zu deuten sind. So fallen besonders einige Kanten oder Wülste auf, die den klobigen Eindruck der Parameren noch verstärken und die man in entsprechender Lage bei beiden Arten finden kann; so etwa ein kantiger, ungefähr in halber Höhe medial verlaufender Querwulst, oder eine schräge Längskante auf der apikalen Hälfte der Parameren. Die Valven unterscheiden sich unter anderem durch ihre verschiedene relative Höhe und Dicke, doch ist ihre Spitze bei beiden Arten deutlich ventrad gebogen.

Aufgrund der besonders auffälligen Bildungen der $A$. arnoldi möchte man diese für die spezialisiertere halten. Beide Arten sind wohl sicher untereinander näher als mit irgendeiner anderen bekannten Biene verwandt, haben aber doch beträchtliche Unterschiede entwickelt. Über die Lebensweise ist fast nichts bekannt.

Nach dieser Übersicht der Merkmale erweist sich eine systematische Einordnung von Aspidosmia als äußerst schwierig. Einigermaßen sicher scheint nur ihre Zugehörigkeit zur Familie Megachilidae zu sein. Vor einer 
weiteren Diskussion muß deshalb die Systematik dieser Gruppe im Gesamtverband der übrigen Apoidea kurz untersucht werden.

\section{C. - ALLgemeines Über PHYLogenetik UND MERKMALSWERTUNG}

Spätestens seit dem Erscheinen der bahnbrechenden Veröffentlichungen Hennigs $(1950,1957,1969)$ dürfte kein Zweifel mehr daran bestehen, daß die Monophylie einer systematischen Gruppe nur angenommen werden kann, wenn die betreffende Gruppe sich durch eine oder mehrere Synapomorphien (gemeinsame abgeleitete Merkmale) auszeichnet. Plesiomorphien (ursprüngliche Merkmalsausprägung) haben in diesem Zusammenhang dagegen keinerlei Beweiskraft. Wie an anderer Stelle ausgeführt wird (Peters und Gutmann, 1971), müßte die Unterscheidung von Apo- und Plesiomorphie auf dem Wege der Rekonstruktion des Anpassungsvorganges geschehen, d.h. durch eine gründliche Konstruktions- und Funktionsanalyse der untersuchten Organismen. Leider ist dies in der Praxis und vor allem in der a Kleinsystematik ", bedingt durch unsere mangelhaften Kenntnisse, oft (noch) nicht möglich. Die Analyse wird in solchen Fällen häufig durch eine bereits vorliegende “ Großsystematik 》 ersetzt, von der man dann nur hoffen kann, daß wenigstens in ihr die Richtung einigermaßen mit den tatsächlichen phylogenetischen Abläufen übereinstimmt. Die Lesrichtung der Großsystematik induziert nämlich die Entscheidung, was in der Kleinsystematik als Apomorphie zu gelten hat und was nicht. Um bei einem banalen Bienen-Beispiel zu bleiben : Nur weil wir zu wissen glauben, daß die nächsten Verwandten der Bienen unter den Grabwespen zu suchen und die "Wespenmerkmale 》 die primitiveren sind, halten wir bei Bienen eine kurze, gespaltene Glossa für primitiv, eine lange, zugespitzte für abgeleitet. Rein theoretisch könnte man es sich ja auch umgekehrt vorstellen. Die Frage wäre jetzt : welche Lesrichtung läßt sich plausibler als Anpassungsweg erklären? Wir wollen unser Beispiel aber nicht weiter ausspinnen, sondern es dabei bewenden lassen, daß unsere erste Annahme tatsächlich wahrscheinlicher ist (vgl. z. B. MaLYSEHV 1968).

Die meisten Apidologen richten sich heute nach dem von Michener (1944, 1965) aufgestellten System der Apoidea (ergänzt von Moure et MicheNER 1955). Es umfaßt die Familien Colletidae, Andrenidae, Halictidae, Mellitidae, Megachilidae, Anthophoridae, Fideliidae und Apidae. Dieses System war kein völlig neuer Entwurf, es bestand vielmehr in seinen wesentlichen Teilen schon bei anderen Autoren (s.z. B. Börner 1919, GrütTe 1935, ŚUSTERA 1958).

Eine Einteilung der Bienen nach Hennigs Prinzip ist bisher jedoch noch nicht versucht worden. So ist es nicht verwunderlich, daß die einzelnen 
Gruppierungen des Bienensystems sich auf Merkmale sehr unterschiedlicher Wertigkeit stützen. Zwar hat Michener in seiner nunmehr schon klassischen Arbeit (1944 : 228-229) eine ansehnliche Liste zusammengestellt, in der sich Merkmale in ihrer ursprünglichen (plesiomorphen) und abgeleiteten (apomorphen) Ausprägung gegenüberstehen, bei der Begründung der systematischen Einheiten wurden dann aber oftmals Merkmale ohne Rücksicht auf ihren Charakter, also auch ohne Rücksicht auf ihre phylogenetische Aussagekraft verwendet. So wird, um nur ein Beispiel zu nennen, als hervorstechendstes Merkmal der Colletidae die gespaltene Zunge betrachtet. Diese Form der Mundwerkzeuge gilt aber bei Bienen als ursprünglich und primitiv. Sie kann also keinesfalls für eine Begründung der Monophylie der Colletidae herhalten. Ebensogut könnte man etwa die Monophylie der Fledermäuse mit der Tatsache zu begründen suchen, daß alle Fledermäuse eine fünffingrige Vorderextremität haben.

Selbstverständlich soll damit nicht behauptet werden, die Colletidae seien keine monophyletische Gruppe. Bei einer umfassenden Revision der Bienen-Systematik (die hier nicht beabsichtigt ist) müßte aber geklärt werden, ob tatsächlich Synapomorphien die Annahme der Monophylie rechtfertigen. Das gilt übrigens nicht nur für die genannte, sondern für alle Gruppen der Apoidea.

\section{D. - DIE PHYLOGENETISCHE SYSTEMATIK DER MEgACHILIDA}

Die Megachiliden gelten allgemein als eine der einheitlichsten Gruppen innerhalb der Apoidea. Doch wird diese Einheitlichkeit auch hier mit einem Gemisch von primitiven und abgeleiteten Merkmalen begründet, wobei manchmal sogar erstere besonders hervorgehoben werden. Manche Autoren (z. B. Michener 1953, Rozen 1970) scheinen das Nebeneinander von sehr ausgeprägt primitiven und deutlich spezialisierten Merkmalen als schwer verständlich empfunden zu haben. Michener hält es deshalb für wahrscheinlich, daß die primitiven Merkmale in Wirklichkeit Reversionen, also nur scheinbar primitiv seien. Doch besteht im Lichte einer phylogenetischen Betrachtung gar kein Grund für eine solche Annahme. Die Schwierigkeiten bestehen für Michener nur deshalb, weil seine Vorstellungen über einen Stammbaum der Apoidea nicht konsequent phylogenetisch sind (Michener 1944 : 236). Die einzelnen Familien bilden bei ihm gewissermaßen eine Stufenleiter, die trotz vieler wichtiger phylogenetischer Implikationen starke typologische Züge aufweist. In einem solchen Stammbaum müssen die einerseits primitiven, andererseits hochspezialisierten Megachiliden selbstverständlich ein Ärgernis sein.

Zweifellos gehören die Megachiliden zu jenen Bienen, die man wegen 
ihrer spezialisierten Mundwerkzeuge mitunter als « höhere Bienen » bezeichnet. Zu den Eigenheiten dieser Mundwerkzeuge gehört vor allem auch die Vförmige Ausprägung des Submentum und das schlanke, sich basad verschmälernde Mentum. Beide Teile spielen in dem komplizierten Faltmechanismus der Mundgliedmaßen eine wichtige Rolle. Michener hat sicher recht (MicheNER 1944 : 227), wenn er es für nahezu unmöglich hält, daß diese Ausformung mehr als einmal entstanden sein sollte. Das heißt, alle Bienen mit diesem Mechanismus können ziemlich sicher als monophyletische Gruppe gelten. Dazu gehören außer den Megachiliden die Apidae, Antophoridae, Fideliidae, Melittidae und die Panurginae. (Da letztere bisher als Unterfamilie der Andrenidae gelten, müßte die Umgrenzung dieser Familie geändert werden, denn die Panurginae erscheinen ja nunmehr mit den “ höheren Bienen » näher verwandt als mit den übrigen Andreniden! Eine Ansicht, die auch Michener schon ausspricht und in seinem Stammbaum darstellt, ohne jedoch die eben genannte Konsequenz zu ziehen.)

Wollen wir die Verwandtschaft der Megachiliden enger einkreisen, so können wir diejenigen Bienen zusammenfassen, die sich durch folgende Synapomorphien auszeichnen (in Klammern sind die jeweiligen Plesiomorphien angegeben; die Ziffern entsprechen denen der Abb. 10).

1. Die ersten zwei Glieder der Labialpalpen sind stark verlängert und flächig ausgewalzt. (Glieder kürzer und \pm zylindrisch).

2. Die Galea ist postpalpal stark verlängert (Galea kurz.).

3. Die Glossa ist stark verlängert (Glossa kurz.).

4. Mittelhüften vergrößert; ihre Länge entspricht etwa dem kürzesten Abstand zwischen ihnen und der Hinterflügelwurzel (Mittelhüfte viel kürzer).

5. Die Mandibeln der Larve haben eine subapikale Grube (Mandibeln ohne Gruben).

Die so umschriebene Gruppe umfaßt die Megachilidae, Fideliidae, Apidae und Anthophoridae. An Versuchen, die phylogenetischen Beziehungen zwischen ihnen und innerhalb dieser Familien zu erkennen, hat es nicht gefehlt, doch steht eine zufriedenstellende Lösung noch aus. Das hängt wohl mit den zahlreichen Überschneidungen der Merkmalskomplexe bei verschiedenen Untergruppen zusammen, so daß man gezwungen ist, sehr häufige Parallelen und Konvergenzen der Stammesentwicklung anzunehmen.

Hinzu kommt, daß wir die anatomischen, physiologischen oder ethologischen Eigenarten der vielen hundert in Frage stehenden Arten nur stichprobenartig kennen. Hat man aber erst einmal eine kleinere Gruppe, etwa eine größere Gattung, genauer untersucht und dabei die vielen überraschenden Unregelmäßigkeiten und Ausnahmen festgestellt, so verzichtet man bald darauf, auf Stichproben Verallgemeinerungen aufzubauen. 


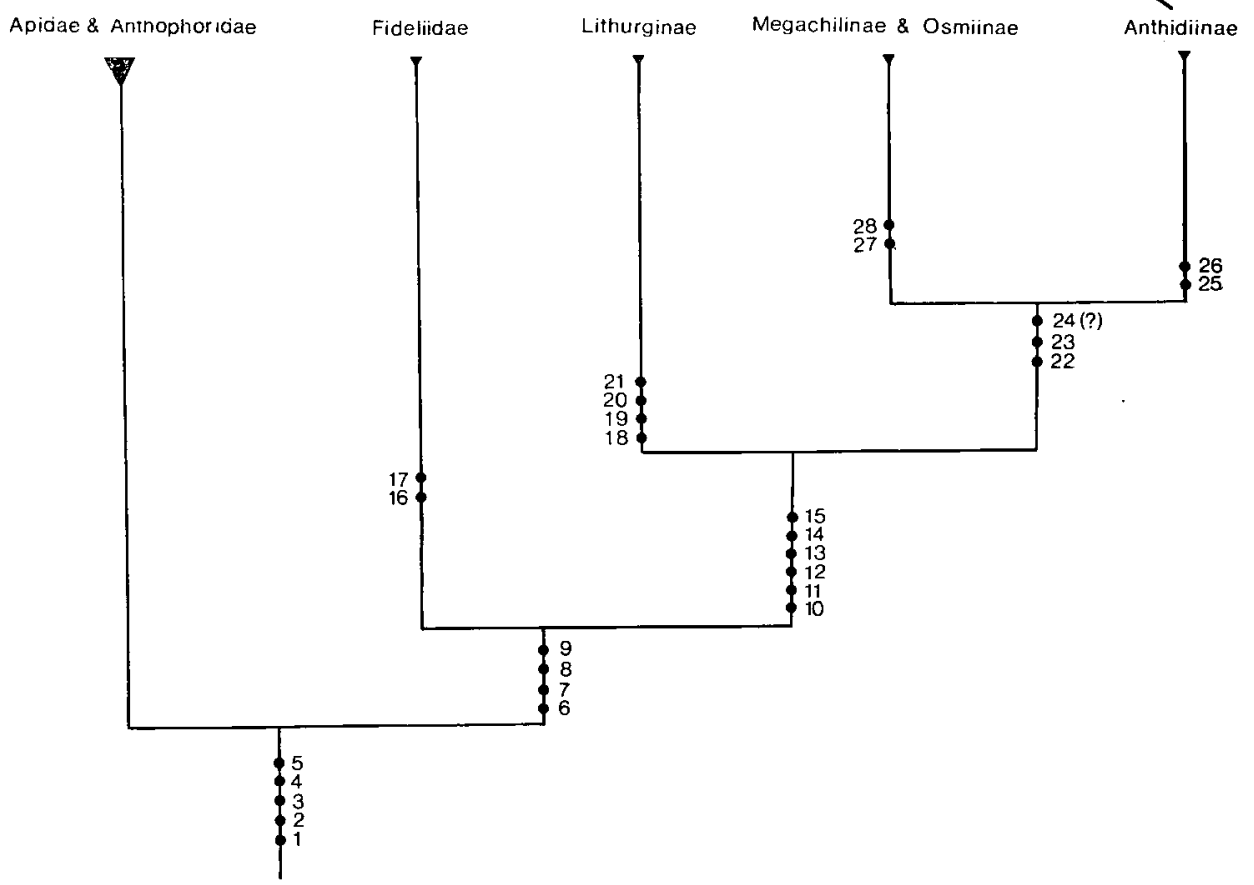

Aвb. 10. - Stammbaum der Megachilidae und ihrer Schwestergruppe. Die Zahlen und Punkte entsprechen den im Text aufgeführten und besprochenen Synapomorphien.

Fig. 10. - Arbre généalogique des Megachilidae et du groupe frère. Les nombres et les points correspondent aux synapomorphies citées et discutées dans le texte.

Folgendes Beispiel möge dies veranschaulichen. Die Rückbildung der Volsellae an den männlichen Genitalien gilt bislang als typisches Merkmal der Megachilidae, im Gegensatz etwa zu den Fideliidae, bei denen die Volsellae noch eine Andeutung der ursprünglichen Spaltung in Cuspis und Digitus zeigen. Bei Untersuehungen an Osmiinae fand ich nun bei einigen Vertretern der Gattungen Noteriades Cockerell 1931 und Pseudoheriades Peters 1970 ebenso gestaltete Volsellae. Diese Befunde legen die Vermutung nahe, daß reduzierte Volsellae gar nicht zur “ Grundausstattung 》 der Megachilidae gehören, sondern daß die Volsellae innerhalb dieser Familie mehrmals parallel einer Rückbildung unterlagen.

Versucht man trotzdem bei der Klärung der verwandtschaftlichen Beziehungen der Megachilidae fortzuschreiten, so bieten sich die Fideliidae für eine nähere Betrachtung an, und zwar zunächst allein deshalb, weil sie mit den Megachiliden eine ausgeprägte Bauchsammelbürste gemeinsam haben.

Nun haben aber die Fideliidae schon manches Kopfzerbrechen verursacht. Popov (1939) stellte sie in die Nähe der Colletidae, da sie mit diesen in der Ausbildung des 7. Sternits der Männchen übereinstimmen. Eine solche Sternitbildung (mit kompliziert geformter, oft lappig zerteilter Apikalhälfte) ist aber, wie Popov selbst bemerkt, auch noch bei anderen Bienen zu finden. In 
abgewandelter Form zeigt sie sich sogar bei verschiedenen Anthophoriden. Deshalb hielt auch GrüTTE (1935) die Sternitbildungen für äußerst unzuverlässige Merkmale, die augenscheinlich häufige Konvergenzen darstellen. Die wahrscheinlichste Deutung scheint mir jedoch zu sein, daß besagte Form des 7. männlichen Sternits zu den ursprünglichen Merkmalen der Bienen überhaupt gehört. Es wäre demnach eine Synapomorphie der Bienen im Verhältnis zu deren Schwestergruppe. Innerhalb der Apoidea wiederum scheint verschiedentlich eine Rückbildung dieses Sternites eingetreten zu sein, oft mit einer teilweisen Übernahme seiner Funktion und der damit einhergehenden komplizierten Verformung durch davorliegende Sternite. Am weitesten ist dieser Prozeß offensichtlich bei den Megachilidae fortgeschritten. Das heißt aber auch, daß innerhalb der Apoidea ein gelapptes 7. Sternit gegenüber einem einfach gebauten als Plesiomorphie zu gelten hat. Aus diesem Grunde wäre dann Popov Beweisführung nicht schlüssig.

Obwohl sie sich der eben ausgeführten Argumentation enthielten, haben Moure et Mrchener (1955), meines Erachtens mit Recht, die in Südamerika entdeckte und ein relativ einfach gebildetes Sternit 7 aufweisende Neofidelia zu den bis dahin nur aus Südafrika bekannten Fideliidae gestellt.

Neuerdings hat Rozen (1970) die Fideliidae aufgrund von Untersuchungen an Larven und Puppen als mit den Megachilidae nahe verwandt bezeichnet. Leider sind viele der von ihm aufgeführten Merkmale Symplesiomorphien der beiden Gruppen und daher für eine nahe Verwandtschaft ohne Beweiskraft (z. B. die relativ starke Behaarung der Larven, deutliche Antennalpapillen, kräftige, zweizähnige Mandibeln usw.).

Dennoch glaube auch ich, in den Fideliidae die Schwestergruppe der Megachilidae sehen zu können, und zwar, weil für beide wenigstens einige Synapomorphien wahrscheinlich zu machen sind, während es sehr schwierig ist, ähnliches für die Megachilidae und irgendeine der Apidae-AnthophoridaeGruppen zu tun. Es handelt sich hierbei um folgende Merkmale (die Nummern entsprechen denen der Abb. 10).

6. Labrum wesentlich länger als breit.

7. Basitibialplatten völlig reduziert.

8. Scopa auf den Sterniten.

9. Zähnelung auf der Innenfläche der larvalen Mandibeln ( c multidentate area or cusp » nach Michener, 1953) stark reduziert.

Es bleibt zu bemerken, daß die Merkmale 7 und 9 auch bei verschiedenen Vertretern der Apidae oder Anthophoridae vorkommen, aber dann offensichtlich konvergent entstanden sind. Das gilt besonders für Merkmal 9. Sollte die vorliegende Interpretation jedoch nicht zutreffen, dann gehören entweder Nomada, Neopasites, Epeolus und Triepeolus nicht zum Apidae-Anthophoridae- 
Komplex oder diese Gattungen haben die Zähnelung sekundär wieder erworben. In jedem Fall ist die hier vertretene Auffassung die ( sparsamste », da sie mit der geringsten Zahl von Neubildungen bereits verlorener Merkmale rechnet.

Unter der Voraussetzung, daß die eben dargestellte Auffassung stimmt, bietet die weitere Aufschlüsselung nur noch geringe Schwierigkeiten. Die Megachilidae erweisen sich als die Gruppe mit den zahlreicheren Synapomorphien (in Klammern die Ausprägung bei den Fideliidae; s. Abb. 10)

10. Subantennalnaht endet außen an der Fühlergrube (Subantennalnaht endet an der Innenseite der Grube).

11. Episternalnaht fehlt (Episternalnaht vorhanden).

12. Epimeralnaht (« scrobal suture 》 nach Michener, 1944) fehlt (diese Naht vorhanden).

13. Vorderflügel mit 2 Submarginalzellen (3 Submarginalzellen).

14. Analplatte stark reduziert (Analplatte gut ausgebildet).

15. Zähnelung auf der Innen fläche der larvalen Mandibeln völlig reduziert (Zähnelung noch als kleiner Rest vorhanden).

Die Fideliidae weisen lediglich zwei Synapomorphien auf, wobei die zweite nur aufgrund von Untersuchungen an einer Gattung (Rozen 1970) angenommen wird.

16. Scopa an den Hinterbeinen als « Sandfeger » ausgebildet und benutzt.

17. “ Behaarung » der Puppe nur noch in Form kurzer Dörnchen vorhanden.

Es mag nicht sehr sinnvoll erscheinen, den « Sandfeger » der Fideliiden als Apomorphie gegenüber den Megachiliden anzuführen, da doch diese an den Hinterbeinen ohne Scopa sein sollen. Gerade Aspidosmia aber zeigt, daß die Megachilidae ursprünglich neben der Bauchscopa wohl auch noch eine Sammelbürste an den Hinterbeinen hatten.

Ansätze zu einer Bauchbürste finden wir bei den Apoidea mehrmals. Der Vorteil einer solcherart vergrößerten Sammeleinrichtung liegt wahrscheinlich in der größeren Pollenmenge, die pro Ausflug ins Nest gebracht werden kann. Solange eine a Bauchbürste 》 nur schwach ausgebildet war, wie etwa bei einigen heutigen Halictiden, mußte natürlich die Beinscopa erhalten bleiben. Das änderte sich aber, als eine wohlausgebildete, leistungsfähige Bauchbürste entstanden war. Da nun dieser Sammelapparat ausreichende Pollenmengen faßte, konnte die Bürste an den Beinen reduziert oder « umfunktioniert » werden. Letzteres führen uns die Fideliidae vor, während die Megachilidae den ersten Weg beschritten. Wahrscheinlich waren die Fideliidae “ von Anfang an 》Wüstenbewohner, die in trockenem Sandboden nisteten, so daß die Funktionen ihrer Beinbürste nahtlos einander ablösen konnten.

Haben wir eben Megachilidae und Fideliidae als Schwestergruppen gegenübergestellt, so ist doch noch eine andere Gruppierung möglich. Wir sagten bereits, daß Neofidelia, der amerikanische « Zweig » der Fideliidae, ein einfach gebildetes männliches Sternit 7 aufweist, das sehr von der komplizierten 
Form bei Fidelia abweicht. Dieses Sternit von Neofidelia sieht dem von Aspidosmia ungemein ähnlich. Träfe es zu, daß diese Sternitform nur einmal entstanden ist, dann müßten die Fideliidae enger begrenzt und Neofidelia noch zu den Megachilidae gezogen werden. Wie sich dann das Apomorphieschema unseres Stammbaumes verändern würde, braucht hier nicht vorgeführt zu werden, da sich die Veränderungen nach den vorliegenden Daten leicht vornehmen lassen. Ob solche Veränderungen tatsächlich nötig sind, müßten künftige Untersuchungen erweisen (z.B. sind die Larven von Neofidelia noch unbekannt). Ich gestehe, daß die Entscheidung, die Fideliidae in ihrer üblichen Zusammensetzung bestehen zu lassen, ein wenig gefühlsmäßig erfolgte. Selbst bei einer Abtrennung von Neofidelia würde sich an der für unsere weiteren Úberlegungen wesentlichen Monophylie der Fideliidae + Megachilidae jedoch nichts ändern.

Versuchen wir schließlich noch die Teilgruppen der Megachilidae durch Synapomorphien zu begründen. Auch hier hat Mrchener richtungsweisende Unterscheidungen getroffen, wenn er auch wiederum oftmals gerade die jeweiligen Plesiomorphien als Beweise anführte, so etwa den großen Jugallobus der Hinterflügel für die Lithurginae. Soweit ich sehe, lassen sich bei den beiden Hauptstämmen folgende Synapomorphien feststellen (Abb. 10) :

18. Hinterschienen auffällig bedornt (Hinterschienen ohne Dörnchen)

19. Gesicht der Weibchen unterhalb der Fühler bucklig vorgewölbt (Gesicht “ normal »)

20. Tergit 1 deutlich verkleinert, mit bogenförmigem Hinterrand (Tergit 1 “ normal », sein Hinterrand \pm gerade)

21. Sternit 7 der Männchen stark rückgebildet (Sternit 7 noch kräftig ausgebildet)

22. Jugallobus des Hinterflügels höchstens von halber Länge des Anallobus (Jugallobus wesentlich länger)

23. Pygidialfeld völlig verschwunden (Pygidialfeld vorhanden, wenn auch reduziert und umgestaltet)

24. Hinterhüfte mit ventralem, scharfem Längsgrat (ein solcher Grat fehlt).

Diese Aufstellung mag manchen Widerspruch herausfordern, so etwa der Punkt 21. Ist doch bei vielen anderen Megachiliden das 7. Sternit ebenfalls stark reduziert. Doch gibt es unter den Anthidiinae und Osmiinae eine ganze Reihe von Arten mit sehr ansehnlichem Sternit 7, so daß man annehmen muß, daß die Reduktion in diesen Entwicklungszweigen in Konvergenz zu den Lithurginae eingetreten ist, aber nicht zur ursprünglichen Grundausstattung gehört. Auch Punkt 24 bedarf einer Erläuterung. Der Carina auf Coxa 3 hat zuerst Michener (1941) großes Gewicht beigemessen, allerdings nur in der Gruppe, die er als Osmiinae zusammenfaßte. Besagte Carina ist aber viel 
weiter verbreitet und tritt auch bei manchen Vertretern der Megachilinae und Anthidiinae auf, scheint dagegen bei den Lithurginae nicht vorzukommen. Man hat nun die Wahl, sich für ein konvergentes Verschwinden oder ein konvergentes Entstehen der Carina bei den Anthidiinae + Osmiinae + Megachilinae zu entscheiden. Deshalb ist in unserem Schema bei diesem Punkt ein Fragezeichen angebracht.

Die Liste der Synapomorphien ließe sich zweifellos noch erweitern, (z.B. zeigen die Lithurginae eine sehr charakteristische Ausformung von Scutellum, Metanotum und Propodeum, die sicher als Synapomorphie dieser Gruppe gedeutet werden muß), doch reichen die genannten Punkte wohl für eine Begründung der beiden Hauptstämme der Megachilidae aus.

Unerwartet große Schwierigkeiten macht dagegen die Trennung von Megachilinae, Osmiinae und Anthidiinae. Die Merkmalskomplexe der für eine Untersuchung meistens allein zur Verfügung stehenden sklerotisierten Körperteile überschneiden sich hier so vielschichtig, daß eine Unterscheidung von Synapomorphie und Parallelentwicklung oft nicht gelingt. Außerdem zeigt es sich, daß die scheinbar so eindeutigen Merkmale in den üblichen Bestimmungsschlüsseln teilweise als Plesiomorphien anzusehen sind. Das gilt zum Beispiel für die gespaltenen Krallen der Weibchen bei den Anthidiinae. Apomorph sind vielmehr die einfachen Krallen, und man darf wohl mit Recht in diesem Merkmal eine schwerwiegende Synapomorphie der Megachilinae \& Osmiinae vermuten. Eine weitere Synapomorphie letzterer Gruppen ist höchstwahrscheinlich in dem Verlust der Randzähnelung der larvalen Mandibeln zu sehen, allerdings fehlen hierzu noch größere Serienuntersuchungen. So stellt sich die Begründung der Monophylie für die Megachilinae und Osmiinae wahrlich nicht imponierend dar. Leider gilt besonders für diese Gruppen, was oben über Stichprobenuntersuchungen gesagt wurde. Die wenigen vorliegenden Ergebnisse der inneren Anatomie, Physiologie, Ethologie usw. lassen kaum Verallgemeinerungen zu. Möglicherweise kann die Zweiteilung des mesalen Remotors der Hinterhüfte als weitere Synapomorphie der Megachilinae \& Osmiinae gelten (WILLE 1956), doch ist dies nicht sicher.

Nicht viel anders liegen die Dinge bei den Anthidiinae. Selbst in neueren Arbeiten über diese Gruppe (Michener 1948, Pastells 1969) gewinnt man zwar einen Überblick über die Vielgestaltigkeit dieser Bienen, findet aber kaum Angaben über allen gemeinsame Merkmale. Außer den bereits erwähnten und nichts beweisenden gespaltenen Krallen wird hier gewöhnlich nur noch das kurze Flügelmal genannt. Dieses Merkmal ist wahrscheinlich eine echte, wenn auch kaum eindeutig zu beweisende Synapomorphie der Anthidiinae; man findet bei keiner Art aus dieser Gruppe so große Flügelmale wie etwa bei primitiven Osmiinae. Als weitere Synapomorphie der Anthidiinae kann die starke Thoraxverkürzung gelten, die den Thorax der Kugelform annähert. Man könnte hier einwenden, daß ähnliche Verkürzungen auch bei anderen 
Megachiliden, etwa bei Osmien, auftreten, doch handelt es sich dabei höchstwahrscheinlich um Parallelentwicklungen. Während bei den näheren Verwandten der Osmien (etwa bei Chelostoma) der Thorax langgestreckt ist, sind bei den Anthidiinae solche ursprüngliche Thoraxbildungen gar nicht zu finden. Daneben sprechen auch einige Unterschiede der Proportionen dafür, daß der kugelförmige Thorax von den Anthidiinae gesondert erworben wurde. Bei ihnen nimmt z. B. das Mittelfeld (“ herzförmiger Raum ») ungefähr die Hälfte der vertikalen Fläche des Propodeum ein. Bei den Megachilinae \& Osmiinae ist der vertikale Teil dieses Feldes wesentlich kleiner und umfaßt etwa ein Drittel der Propodeumrückwand. Nicht im Widerspruch zu der Behauptung einer synapomorphen Thoraxverkürzung der Anthidiinae steht die bemerkenswerte Tatsache, daß auch bei den Anthidiinae Arten auftreten, deren Propodeum ähnlich wie bei Heriades einen grob gerippten horizontalen Teil aufweist. Dieser horizontale Teil hat mit allergrößter Wahrscheinlichkeit nichts mit den horizontalen Propodeumflächen ursprünglicher Formen zu tun, sondern ist eine sekundäre Ausbildung. Der genannten Propodeumbidung entspricht meistens ein vorn breit konkaves und mit einer Querkante versehenes erstes Tergit. Es steht außer Zweifel, daß diese Bildungen bei den Osmiinae mehrmals entstanden sind, wahrscheinlich im Zusammenhang mit einer noch unbekannten, von der Lebensweise geforderten Funktion. Es mag kein Zufall sein, daß diejenigen Anthidiinae mit der stärksten Ausprägung einer solchen Propodeumbildung im tropischen Amerika leben, also dort, wo Osmiinae nicht vorkommen.

Hinzugefügt werden muß noch, daß hier die Dioxyni zu den Anthidiinae gezählt werden. Ihre Unterbringung verursacht zwar einige Schwierigkeiten, doch ist nicht zu erkennen, welcher Gruppe man sie besser anschließen könnte (vgl. Grütte 1935).

Damit dürfen wir nun, wenn auch nicht sehr befriedigend, so doch mit einiger Berechtigung folgende Synapomorphien für unseren Stammbaum festhalten :

25. Flügelstigma im Innenrand höchstens so lang wie seine größte Breite (Flügelstigma länger).

26. Thorax annähernd kugelförmig mit steil abfallendem Metanotum und Propodeum (Thorax gestreckter, Metanotum auf seiner Horizontalfläche, Propodeum mit horizontalem Basisteil).

27. Klauen der Weibchen einfach (Klauen gespalten).

28. Die Randzähnelung der larvalen Mandibeln rückgebildet (Larvale Mandibeln gezähnelt).

Abschließend zu diesem Kapitel sei noch bemerkt, daß die Unterfamilien der Megachilidae, so wie sie hier benannt werden, stammesgeschichtlich nicht gleichwertig sind. Ein Blick auf den Stammbaum zeigt, daß Megachilinae, Osmiinae und Anthidiinae zusammen erst den Rang der Lithurginae haben, 
ohne daß dies in der hier verwendeten Nomenklatur sich ausdrücken würde. Michener (1944) hat den ersten drei Gruppen nur Tribus-Rang zuerkannt und alle gemeinsam als Unterfamilie Megachilinae bezeichnet. Pasteels (1969) gab den Anthidiinae jedoch den Rang einer Unterfamilie. Dieser Auffassung habe ich mich angeschlossen und bezeichne deshalb auch die beiden anderen Gruppen als Unterfamilien. Betont sei aber, daß dies allein praktische und keine prinzipiellen Gründe hat. In einem phylogenetischen System ist ohnehin der Stammbaum das Wesentliche und somit wichtiger als die lediglich dazuerfundenen Namen (Peters 1970).

\section{E. - DIE STELLUNG VON ASPIDOSMIA}

Die vorausgegangene Analyse hat über Aspidosmia nur teilweise Klarheit geschaffen. Der größte Teil der für diese Gattung kennzeichnenden Merkmale erweist sich als plesiomorph. Immerhin läßt sich sagen, daß sie sicher nicht zu den Lithurginae gehört. Zu den Megachilinae-Osmiinae kann sie ebenfalls nicht gehören, da ihr deren Synapomorphie « einfache Klauen » fehlt. Dagegen weist sie beide Synapomorphien der Anthidiinae auf : ein relativ kurzes Flügelmal und einen verkürzten Thorax mit großem Mittelfeld des Propodeum. Es erseheint mir deshalb richtig, Aspidosmia bei den Anthidiinae einzureihen. $\mathrm{Da}$ es einen genealogischen Stammbaum der Anthidiinae noch nicht gibt, fällt eine weitergehende Eingliederung von Aspidosmia jedoch schwer. Manches spricht dafür, daß Aspidosmia allen übrigen Anthidiinae gegenübersteht; zu erwähnen wären etwa die mutmaßliche Sammelbürste der Hinterbeine, der gebogene Basalnerv, die undifferenzierte Flügelbehaarung, der lange Jugallappen, die Arolia, die langen Strigilis-Spitzen. Freilich gibt es auch andere Anthidiinae mit dem einen oder anderen dieser plesiomorphen Merkmale, doch bei keinem wohl treten sie so gehäuft auf. Als Synapomorphien der beiden Aspidosmia-Arten hätten zu gelten : die borstenartige Behaarung des Hinterleibsendes; die glatten, eingedrückten Tergitränder; die Skulptur des Propodeum; Ausmaß und Form des gelben Fleckes auf dem Clypeus der Männchen. Die Gelbzeichnung an sich ist eher als ursprünglich einzustufen, und zwar gilt dies wohl für die Apoidea schlechthin.

Für die Systematik liegt die Bedeutung von Aspidosmia vor allem darin, daß man mit Hilfe dieser Gattung das Bild der Biene ergänzen kann, die als “ Ur-Megachilide » an der Wurzel der Familie Megachilidae zu vermuten ist. Diese Biene muß folgende Merkmale gehabt haben : Die wohlausgebildeten Mundwerkzeuge der « höheren Bienen », Labrum länger als breit, Subantennalnaht am Außenrand der Fühlergrube endend, Clypeus beim Männchen gelb, Clypeus die Labrumbasis nicht überragend, Parapsidalfurchen linienförmig, Metanotum und Basisteil des Propodeum in der Horizontalfäche des Thorax, 
alle Pleuralnähte fehlen, Klauen auch beim Weibchen gespalten, Arolia vorhanden, Strigilis mit langer Endspitze, Hinterschienen und Sternite der Weibchen mit Scopa, Flügel ziemlich gleichmäßig behaart, Flügelmal groß, 2 Submarginalzellen vorhanden, beide rücklaufenden Nerven münden in die zweite Submarginalzelle, Jugallappen des Hinterflügels über halb so lang wie der Anallappen, Tergit 1 mit langer Seitennaht, Analplatte vorhanden, beim Männchen sechs Sternite sichtbar, Sternit 7 mit \pm dreispitzigem Endrand, die beiden Außenspitzen länger und am Ende behaart, Sternit 8 im Grundtriß annähernd rautenförmig und am Endrand behaart, Larven behaart, mit zweizähnigen Mandibeln u. großen Antennalpapillen, Mandibeln mit apikaler Grube, aber ohne Zähnelung auf der Innenfläche, dagegen Zähnelung am Rand, Öffnung der larvalen Speichelgänge groß, schlitzförmig, mit deutlichen « Lippen ", Larve spinnt festen Kokon.

Damit liegt ein (vorläufiger ?) Bezugspunkt fest, an dem das Puzzlespiel (Hennig 1950) der Aufdröselung phylogenetischer Zusammenhänge der Megachilidae bei künftigen Untersuchungen anknüpfen kann.

Eingegangen im Februar 1972. Reçu pour publication en février 1972.

\section{DANKSAGUNG}

Die vorliegende Untersuchung war nur möglich durch die Ausleihe von Typen und anderem Material aus verschiedenen Sammlungen. Ich danke herzlich für die erwiesene Hilfe folgenden Institutionen : Albany Museum, Grahamstown (durch Vermittlung von Herrn Prof. Dr. J.-J. Pasteers, Bruxelles); American Museum of Natural History (Dr. J.-G. Rozen jr.); National Museum of Southern Rhodesia, Pretoria (durch Vermittlung von Herrn Prof. Dr. J.-J. PAsteers, Bruxelles); South African Museum, Cape Town (Dr. A.-J. Hesse); Transvaal Museum (S. W. L. de Kock, Dr. L. VAri, Dr. J.-A. van Reenen); Zoologisches Museum, Berlin (Dr. E. Königsmann).

\section{RESUME}

1. Lorsque Brauns décrivit une abeille sud-africaine comme Aspidosmia, il pensa devoir la considérer comme un sous-genre d'Osmia. Les présentes recherches montrent qu'Aspidosmia ne peut pas appartenir à Osmia du fait qu'elle montre plutôt des synapomorphies avec les Anthidiinae. A l'intérieur de ce groupe elle se trouve vis-à-vis de tous les autres genres; elle montre de nombreux caractères primitifs en premier lieu une brosse manifestement encore fonctionnelle sur les tibias postérieurs, bien qu'une brosse ventrale soit également présente. On peut donc supposer que la forme originelle des Megachilidae possédait également les deux dispositifs de récolte l'un à côté de l'autre. Une caractérisation hypothétique de cette forme originelle constitue la dernière partie du présent article.

Cependant que jusqu'ici pour Aspidosmia seule l'espèce $A$. arnoldi Brauns 1926 était connue (elle doit être considérée comme l'espèce-type du genre), Osmia volkmanni FrIESE 1909 a été reconnue maintenant comme appartenant aussi aux Aspidosmia. Le mâle de cette espèce 
a été décrit comme Osmia grobbelaari Brauns 1926. Les mâles d'arnoldi et volkmanni se ressemblent beaucoup. Du fait que les deseriptions de Brauns et Friese ne contiennent pas certains détails importants (par exemple : griffes fourchues des femelles, clypeus des mâles marqué de jaune), on donne ici une description plus exacte des deux espèces. Jusqu'ici on ne connaît que les imagos; les données sur la biologie manquent presque complètement. Les deux espèces semblent n'exister qu'en Afrique du Sud et être rares.

2. Pour interpréter correctement les particularités d'Aspidosmia, une analyse des caractères de la famille des Megachilidae était nécessaire. Le procédé se basait dans ce cas sur le principe de Henning de la recherche des groupes frères. La classification des Megachilidae précédemment établie par Michener a pu être confirmée. Comme groupe frère des Megachilidae les Fideliidae sont à considérer. Le résultat de la discussion phylogénétique est représenté par la figure 10. Les numéros se rapportent aux synapomorphies suivantes :

1. Les deux premiers articles des palpes labiaux sont fortement allongés et aplatis.

2. La galea est fortement allongée après le palpe.

3. La glosse est fortement allongée.

4. Hanches moyennes agrandies; leur longueur correspond environ à l'espace le plus court entre elles et la racine de l'aile postérieure.

5. Les mandibules de la larve ont une fosse sub-apicale.

6. Labre sensiblement plus long que large.

7. Plaques basi-tibiales complètement réduites.

8. Brosse sur les sternites.

9. Dentelure de la face interne de la mandibule larvaire (“ multidentale area or cusp » selon Michener, 1953) fortement réduite.

10. Suture sub-antennaire se terminant extérieurement sur la fossette antennaire.

11. La suture épisternale manque.

12. La suture épimérale (“ scrobal suture » selon Michener, 1944) manque.

13. Aile antérieure avec 2 cellules sub-marginales.

14. Plaque anale fortement réduite.

15. Dentelure sur la face interne de la mandibule larvaire complètement réduite.

16. Brosse sur les pattes postérieures formée et utilisée comme un « balai ».

17. “Pilosité » de la nymphe n'existant plus que sous la forme de courtes épines.

18. Tibias postérieurs nettement épineux.

19. Face de la femelle au-dessous des antennes bossues.

20. Tergite 1 nettement réduit, avec bord postérieur arqué.

21. Sternite 7 des mâles fortement régressé.

22. Lobe jugal de l'aile postérieure au plus de la moitié de la longueur du lobe anal.

23. Plaque anale complètement disparue.

24. Hanche postérieure présentant une arête ventrale aigüe dans le sens de la longueur.

25. Ptérostigma sur le bord interne tout au plus aussi long que sa plus grande largeur.

26. Thorax approximativement sphérique avec métanotum et propodeum tombant à pic.

27. Griffes des femelles non fourchues.

28. Dentelure marginale de la mandibule larvaire régressée. 


\section{LITERAT URVERZEICHNIS}

Börner C., 1919. Stammesgeschichte der Hautflügler. Biol. Zbl. 39, 145-186.

Brauns H., 1926. V. Nachtrag zu “ Friese, Bienen Afrikas 》. Zool. Jb., Syst. 52, 187-230.

GrütTe E., 1935. Zur Abstammung der Kuckucksbienen. Z. wiss. Zool., Abt. B.N.F. 4, 449-534.

Hennig W., 1950. Grundzüge einer Theorie der phylogenetischen Systematik. Deutscher Zentralverlag, Berlin.

Hennig W., 1957. Systematik und Phylogenese. Ber. Hundertjahrfeier dtsch. ent. Ges. Berlin, 50-71.

Hennig W., 1969. Die Stammesgeschichte der Insekten. Verlag Waldemar Kramer, Frankfurt M.

Malyshev S. J., 1968. Genesis of the Hymenoptera and the Phases of their Evolution. Methuen, London.

Michener Ch. D., 1941. A Generic Revision of the American Osmiinae with Descriptive Notes on Old World Genera. Amer. Midl. Natur. 26, 147-167.

Michener Ch. D., 1944. Comparative external morphology, phylogeny, and a classification of the bees (Hymenoptera). Bull. am. Mus. natur. Hist., 82, 156-326.

Michener CH. D., 1948. The Generic Classification of the Anthidiine Bees (Hymenoptera, Megachilidae). Am. Mus. Nov., Nr. 1381, 1-28.

Michener Ch. D., 1953. Comparative Morphological and Systematic Studies of Bee Larvae with a Key to the Families of Hymenopterous Larvae. Univ. Kansas Sc. Bull. 35, 987-1102.

Mrchener Ch. D., 1965. A Classification of the Bees of the Australian and South Pacific Regions. Bull. amer. Mus. natur. Hist. 130, 1-362.

Moure J. S. und Ch. D. Michener, 1955. The Bee Family Fideliidae in South America (Hymenoptera, Apoidea). Dusenia, 6, 199-206.

Pasteels J. J., 1969. La systématique génerique et subgénerique des Anthidiinae (Hymenoptera, Apoidea, Megachilidae) de l'Ancien Monde. Mem. Soc. roy. d'Entom. Belgique, $31,1-148$.

Peters D S., 1970. Über den Zusammenhang von biologischem Artbegriff und phylogenetischer Systematik. Aufs. u. Reden d. Senckenbergischen Naturf. Ges. 18, 1-39.

Peters D. S. und W. F. GutmanN, 1971. Über die Lesrichtung von Merkmals- und Konstruktionsreihen. Z. f. zool. Syst. u. Evolutionsf. 9, 237-263.

Popov V. V., 1939. Family Fideliidae and Morphological Convergence among Bees (Apoidea). Comptes Rend. de l'Acad. Sc. U.R.S.S., 22, 640-643.

Rozen J. G. jr., 1970. Biology, Immature Stages, and Phylogenetic Relationships of Fideliine Bees, with the Description of a New Species of Neofidelia (Hymenoptera, Apoidea). Amer. Mus. Nov., Nr. 2427, 1-25.

Snodgrass R. E., 1941. The Male Genitalia of Hymenoptera. Smithson. miscell. Coll., 99, (14), $1-86$.

ŠUSterA Q., 1958. Übersicht des Systems der paläarktischen und mitteleuropäischen Gattungen der Superfamilie Apoidea (Hymenoptera). Acta entom. Mus. nat. Pragae, 32, 443-463.

WILLE A., 1956. Comparative Studies of the Thoracic Musculature of Bees. Univ. Kansas Sc. Bull., 38, 436-499. 